\title{
Gravity Effects on Hawking Radiation from Charged Black Strings in Rastall Theory
}

\author{
Riasat Ali, ${ }^{1, *}$ Rimsha Babar, ${ }^{2,+}$ Muhammad Asgher, ${ }^{3, \ddagger}$ and Syed Asif Ali Shah ${ }^{4, \S}$ \\ ${ }^{1}$ Department of Mathematics, GC University Faisalabad Layyah Campus, Layyah-31200, Pakistan \\ ${ }^{2}$ Division of Science and Technology, University of Education, Township, Lahore-54590, Pakistan \\ ${ }^{3}$ Department of Mathematics, The Islamia University of Bahawalpur, Bahawalpur-63100, Pakistan \\ ${ }^{4}$ Department of Mathematics and Statistics, The University of Lahore 1-Km Raiwind Road, Sultan Town Lahore 54000, Pakistan
}

(Dated: July 27, 2021)

\begin{abstract}
The Rastall theory of gravity is the generalized form of the Einstein theory which describes the conservation law of energy and momentum tensor. In our work, we compute the charged black strings solution in the background of Rastall theory by applying the Newman-Janis approach. After computing the charged black strings solution in the background of Rastall theory, we study the thermodynamical property (i.e., Hawking temperature) for the charged black strings. Furthermore, we investigate the graphical representation of Hawking temperature via event horizon to check the stability conditions of charged black strings under the influence of Rastall theory. Moreover, we examine the modified Hawking temperature for charged black strings in Rastall theory by taking into account the quantum gravity effects. We also discuss the physical state of charged black strings under the effects of quantum gravity and spin parameter (appears due to Rastall theory in charged black strings solution).
\end{abstract}

Keywords: Black strings; Rastall theory; Newman-Janis algorithm; Hawking temperature.

\section{INTRODUCTION}

General relativity (GR) theory of Einstein, which is assumed to be the most interesting and simplest gravity theory, obeys the conservation law of energy-momentum tensor. Although, since its establishment researchers are looking for different gravity theories and several modified gravity theories have been developed. In this campaign, Rastall [1, 2] introduced a very interesting potential modification of GR theory, which does not obey the standard conservation law of energy-momentum tensor (i.e., $T_{; j}^{u v}=0$ ). However, a non-minimal coupling of matter field via space-time geometry can be introduced in the form

$$
T_{u ; v}^{v}=\lambda R_{, u}
$$

here $\lambda$ represents the coupling parameter and describes the deviation from GR. The spherically symmetric static charged as well as uncharged black hole (BH) metric in the context of perfect fluid surrounded by Rastall gravity theory have been analyzed [3]. Additionally, some interest has been committed to provide the static spherically symmetric solutions of the gravitational field equations in the background of Rastall gravity which incorporates the $\mathrm{BH}$, wormholes and neutron star solutions $[4,5]$. The Reissner-Nordström BH metric solution with cosmological constant in Rastall gravity theory has been studied [6].

Spallucci and Smailagic [7] have analyzed the regular BH solution in the context of Rastall gravity theory. They conclude that a regular BH solution exists with exotic matter and have no singularity in General Relativity. The $\mathrm{BH}$ solutions (in the background of perfect fluid matter of rotating $\mathrm{BHs}$ ) in the Rastall theory have been analyzed $[8,9]$. The spherically symmetric and static regular BH metric in the generalized Rastall gravity theory have been investigated [10]. Moreover, the electromagnetic neutral BHs solution and their general properties have also been analyzed.

The theory of Rastall gravity is a generalized gravity theory and also studied the coupling between geometry and matter. According to Visser, the Rastall theory of gravity is equivalent to Einstein gravity [11] but the Darabi and his colleagues [12] conclusion is different from Visser's idea. They proposed that Rastall gravity is not equivalent to Einstein gravity. The rotating BH solution by utilizing Demiański-Newman-Janis algorithm to the electrically charged BH surrounded by quintessence parameter in Rastall gravity theory have been analyzed [13]. Furthermore,

\footnotetext{
*Electronic address: riasatyasin@gmail.com

${ }^{\dagger}$ Electronic address: rimsha.babar10@gmail.com

‡Electronic address: m.asgher145@gmail.com

§Electronic address: asifalishah695@gmail.com
} 
the $\mathrm{BH}$ mass and thermodynamical properties (Hawking temperature, heat capacity and electromagnetic potential) from the horizon equation have also been examined.

Moradpour et al. have analyzed the conformally flat BH solutions in the Rastall gravity as well as non-singular $\mathrm{BH}$ solutions in the background of modified Rastall gravity [14]. The Hawking radiation depends on BH geometry and for different types of particles, we arrive at the same result. Yale [15] have studied the Hawking temperature for every type of particle fermions, scalars and bosons spin-1, by utilizing the tunneling method. The Hawking temperature for symmetric BHs can be derived [15] from the following formula

$$
T_{H}=\frac{f\left(r_{+}\right)}{4 \pi} .
$$

In order to calculate the Hawking temperature, the Hawking radiation phenomenon for different $\mathrm{BHs}$ have been investigated [16]-[40]. Moreover, they have also studied the Hawking temperature for various types of BHs by taking into account the quantum gravity effects. By considering the generalized uncertainty principle (GUP) effects, it is feasible to study the quantum corrected thermodynamical properties of $\mathrm{BH}$ [41]. The GUP offers high-energy remedies to $\mathrm{BH}$ thermodynamics, which guides to the possibility of a minimal length in quantum theory of gravity. The modified fundamental commutation relation can be describes as $\left[x_{\mu}, p_{\mu}\right]=i \hbar \delta_{\mu v}\left[1+\alpha p^{2}\right][42]$.

The expression of GUP can be defined as

$$
\Delta x \Delta p \geq \frac{\hbar}{2}\left[1+\alpha(\Delta p)^{2}\right]
$$

where $\alpha=\frac{\alpha_{0}}{M_{p}^{2}}, \alpha_{0}$ denotes the dimensionless parameter and $\alpha_{0}<10^{5}$, the $M_{p}^{2}$ gives the Plank mass. The $x_{\mu}$ and $p_{\mu}$ denotes the modified position and momentum operators, respectively, which can be given as $x_{\mu}=x_{0 \mu}, p_{\mu}=$ $p_{0 \mu}\left(1+\alpha p_{0 \mu}^{2}\right)$, where $p_{0 \mu}$ and $x_{0 \mu}$ are standard momentum and position operators, respectively, which satisfy the standard commutation relation $\left[x_{0 \mu}, p_{0 \mu}\right]=i \hbar \delta_{\mu \nu}$. We choose the values of $\alpha$ according to the condition, which satisfies the condition of GUP relation [43]. For corrected Hawking temperature, we have choose the only first order terms of $\alpha$ in our calculation. The idea of GUP has been utilized for various BHs in literature [44]-[48].

The main aim of this article is to study the charged black strings solution in the background of Rastall theory and to compare our results with previous literature. This paper is arranged in the following way: In Sec. II, we derive a charged black strings solution in the context of Rastall theory and also investigate the Hawking temperature for the charged black strings. Section III provides the graphical explanation of Hawking temperature via event horizon and states the stability condition of charged black strings under the Rastall theory effects. Section IV analyze the modified Hawking temperature for charged black strings in the Rastall theory. Section $\mathbf{V}$ discusses the effects of quantum gravity and Rastall parameter on charged black strings with the help of graphical interpretation. Finally, Sec. VI consists of summary and conclusions.

\section{CHARGED BLACK STRINGS SOLUTION IN THE CONTEXT OF RASTALL THEORY}

By applying the Demiański-Newman-Janis algorithm, the spin or rotation parameter $a$ can be derived into a spherically symmetric solution which provides an extension for Newman-Janis algorithm. The Rastall gravity depends upon the Rastall theory that established the conservation law of energy-momentum to the accompanying framework as described in Eq. (1). According to this theory, the modified Einstein field equation can be defined as [49]

$$
G_{u v}+\kappa \lambda g_{u v}=\kappa \tilde{T}_{u v},
$$

where $G_{N}$ stands for gravitational constant for the Newton gravity, $\kappa=8 \pi G_{N}$ represents the gravitational constant of the Rastall gravity.

Here, we derive a metric for charged static and stationary black strings in the background of Rastall theory by considering the Newman-Janis algorithm. Moreover, we investigate the Hawking temperature for the corresponding BH metric. For this purpose, we consider the charged static black strings solution [50]

$$
d s^{2}=-F(r) d t^{2}+\frac{1}{F(r)} d r^{2}+r^{2} d \theta^{2}+r^{2} \beta^{2} d y^{2},
$$

where

$$
F(r)=\beta^{2} r^{2}-\frac{b}{\beta r}+\frac{c^{2}}{\beta^{2} r^{2}}
$$


and

$$
\beta^{2}=-\frac{\bigwedge}{3}, \quad b=4 M G, \quad c^{2}=4 q^{2} G,
$$

here the parameters $M$ and $q$ shows the ADM mass per unit length and black string charge, respectively. Moreover, $\wedge$ denotes the cosmological constant as well as $b$ and $c$ represents the arbitrary parameters.

After putting $\beta^{2} r^{2}-\frac{b}{\beta r}+\frac{c^{2}}{\beta^{2} r^{2}}=0$, we can evaluate the event horizon in the given form [51]

$$
r_{+}=\frac{S^{\frac{1}{2}} b^{\frac{1}{3}}+2^{\frac{1}{2}}\left[S^{2}-4 p^{2}-S\right]^{\frac{1}{4}}}{2 \beta}
$$

where

$$
\begin{aligned}
S & =\left[0.5+0.5\left(1-\frac{256 p^{6}}{27}\right)^{\frac{1}{2}}\right]^{\frac{1}{3}}+\left[0.5-0.5\left(1-\frac{256 p^{6}}{27}\right)^{\frac{1}{2}}\right]^{\frac{1}{3}}, \\
p^{2} & =\frac{1}{b^{\frac{4}{3}}} c^{2} .
\end{aligned}
$$

In order to analyze the charged black strings solution in the context of Rastall theory. Firstly, we consider a transformation for black strings metric Eq. (5) from coordinates $(t, r, \theta, y)$ to $(u, r, \theta, y)$ as

$$
d u=d t-\frac{d r}{F(r)}
$$

under the given transformation the Eq. (5) can be defined as

$$
d s^{2}=-F(r) d u^{2}-2 d u d r+r^{2} d \theta^{2}+r^{2} \beta^{2} d y^{2} .
$$

The components of the inverse metric can be given as

$$
g^{u r}=g^{r u}=-1, g^{r r}=F, g^{\theta \theta}=\frac{1}{r^{2}}, g^{y y}=\frac{1}{r^{2} \beta^{2}} .
$$

The inverse metric in the frame of null tetrad can be expressed as

$$
g^{\mu \nu}=-l^{v} n^{\mu}-l^{\mu} n^{v}+m^{\mu} \bar{m}^{v}+m^{v} \bar{m}^{\mu} .
$$

The corresponding elements for null tetrad can be defined in the form

$$
\begin{aligned}
l^{\mu} & =\delta_{r}^{\mu}, \quad n^{\mu}=\delta_{u}^{\mu}-\frac{1}{2} F \delta_{r}^{\mu}, \\
m^{\mu} & =\frac{1}{\sqrt{2} r} \delta_{\theta}^{\mu}+\frac{i}{\sqrt{2} r \beta} \delta_{y}^{\mu}, \\
\bar{m}^{\mu} & =\frac{1}{\sqrt{2} r} \delta_{\theta}^{\mu}-\frac{i}{\sqrt{2} r \beta} \delta_{y}^{\mu},
\end{aligned}
$$

At any point in the black string metric, the relations between the null tetrad and the null vectors becomes

$$
l_{\mu} l^{\mu}=n_{\mu} n^{\mu}=m_{\mu} m^{\mu}=l_{\mu} m^{\mu}=m_{\mu} m^{\mu}=0,
$$

and

$$
l_{\mu} n^{\mu}=-m_{\mu} \bar{m}^{\mu}=1 \text {. }
$$

In the $(u, r)$ plane, the coordinate transformation can be defined as

$$
\begin{aligned}
& u \rightarrow u-i a \cos \theta, \\
& r \rightarrow r+i a \cos \theta,
\end{aligned}
$$


Moreover, we analyze the following transformations

$$
F(r) \rightarrow f(r, a, \theta)
$$

and

$$
r^{2}+a^{2} \cos ^{2} \theta=\Sigma^{2}
$$

In the $(u, r)$ plan the null vectors get the form

$$
\begin{aligned}
l^{\mu} & =\delta_{r}^{\mu}, \quad n^{\mu}=\delta_{u}^{\mu}-\frac{1}{2} f \delta_{r}^{\mu}, \\
m^{\mu} & =\frac{1}{\sqrt{2} r}\left(\delta_{\theta}^{\mu}+i a \beta\left(\delta_{u}^{\mu}-\delta_{r}^{\mu}\right)+\frac{i}{\beta} \delta_{y}^{\mu}\right), \\
\bar{m}^{\mu} & =\frac{1}{\sqrt{2} r}\left(\delta_{\theta}^{\mu}-i a \beta\left(\delta_{u}^{\mu}-\delta_{r}^{\mu}\right)-\frac{i}{\beta} \delta_{y}^{\mu}\right) .
\end{aligned}
$$

According to the null tetrad definition, the non-zero components of inverse metric $g^{\mu r}$ in the $(u, r, \theta, y)$ coordinates can be derived as

$$
\begin{aligned}
& g^{u u}=\frac{a^{2} \beta^{2}}{\sum^{2}}, \quad g^{u r}=g^{r u}=-1-\frac{a^{2} \beta^{2}}{\sum^{2}}, \quad g^{r r}=f(r, \theta)+\frac{a^{2} \beta^{2}}{\sum^{2}} \\
& g^{y y}=\frac{1}{\sum^{2} \beta^{2}}, \quad g^{u y}=g^{y u}=\frac{a}{\sum^{2}}, \quad g^{r y}=g^{y r}=-\frac{a}{\sum^{2}}, \quad g^{\theta \theta}=\frac{1}{\sum^{2}}
\end{aligned}
$$

here

$$
f(r, \theta)=\frac{\beta^{2} r^{4}-\frac{4 M r}{\beta}+\frac{4 q^{2}}{\beta^{2}}}{\Sigma^{2}} .
$$

Furthermore, we analyze a coordinate transformation from $(u, r, \theta, y)$ to $(t, r, \theta, y)$ coordinates in the given form

$$
d u=d t+\Lambda(r) d r, \quad d y=d y+h(r) d r
$$

here

$$
\begin{aligned}
& \Lambda(r)=\frac{r^{2}+a^{2}}{r^{2} F+a^{2}}, \\
& h(r)=-\frac{a}{r^{2} F+a^{2}} .
\end{aligned}
$$

Finally, we compute the black strings metric in the background of Rastall theory under $(t, r, \theta, y)$ coordinates in the following form

$$
\begin{aligned}
d s^{2} & =-\left(\frac{\beta^{2} r^{4}-\frac{4 M r}{\beta}+\frac{4 q^{2}}{\beta^{2}}}{\Sigma^{2}}\right) d t^{2}-2 a \beta^{2}\left(1-\frac{\beta^{2} r^{4}-\frac{4 M r}{\beta}+\frac{4 q^{2}}{\beta^{2}}}{\Sigma^{2}}\right) d t d y+\frac{\Sigma^{2}}{\Delta_{r}} d r^{2} \\
& +\Sigma^{2} d \theta^{2}+\frac{a^{2}\left[\Sigma^{4}+a^{2}\left(-4 q^{2}+\left(4 M r-r^{4} \beta^{3}+2 \beta \Sigma^{2}\right) \beta\right)\right]}{\Sigma^{2}} d y^{2},
\end{aligned}
$$

where

$$
\Delta_{r}=\beta^{2} r^{4}-\frac{4 M r}{\beta}+\frac{4 q^{2}}{\beta^{2}}
$$

The generalized formula for the Hawking temperature has been commonly computed in the previous literature. By using Eq. (2) the Hawking temperature can be evaluated in the following expression

$$
T_{H}=\frac{2 \beta^{4} r_{+}^{5}+4 M \beta r_{+}^{2}-8 r_{+} q^{2}+a^{2}\left(4 \beta^{4} r_{+}^{3}-4 M \beta\right)}{4 \pi \beta^{2}\left(r_{+}^{2}+a^{2}\right)^{2}} .
$$


The temperature $T_{H}$ depends on cosmological constant $\Lambda$ (i. e., $\beta=-\Lambda / 3$ ), spin parameter $a$, black string mass $M$ and black string charge $q$. It is worth mentioning here that for $a=0$, we recover the Hawking temperature for charged black strings [50], which is independent of the spin parameter.

$$
T_{H}=\frac{1}{4 \pi}\left[2 \beta^{2} r_{+}+\frac{4 M}{\beta r_{+}^{2}}-\frac{8 q^{2}}{\beta^{2} r_{+}^{3}}\right] .
$$

\section{GRAPHICAL ANALYSIS}

This section analyzes the graphical explanation of $T_{H}$ w.r.t horizon $r_{+}$. We observe the physical importance of the graphs under the influence of spin parameter and study the stability analysis of corresponding charged black strings. According to Hawking's phenomenon when the temperature increases and more radiations emit then radius of $\mathrm{BH}$ reduces. This physical phenomenon depicts the $\mathrm{BH}$ stability.

In Fig. 1: (i) represents the behavior of $T_{H}$ for fixed $M=100, \beta=-0.001, a=9$ and varying values of $\mathrm{BH}$ charge $q$. It is to be noted that the temperature $T_{H}$ slowly decreases with the increasing values of $r_{+}$in the range $0 \leq r_{+} \leq 8$. This behavior shows the stability of $\mathrm{BH}$.

In (ii), one can observe the behavior of $T_{H}$ for fixed $M=200, \beta=-0.0005, q=0.1$ and varying values of spin parameter $a$. Here, we can see that the $T_{H}$ exponentially decreases as $r_{+}$increases. Moreover, it can be also seen that in the range $3.1<r_{+}<3.3$, the temperature remains same for the various values of $a$. The physical behavior of $T_{H}$ in the range $0 \leq r_{+} \leq 5$ guarantee the stable condition of $\mathrm{BH}$.

(i) $M=100, \beta=-0.001, a=9$

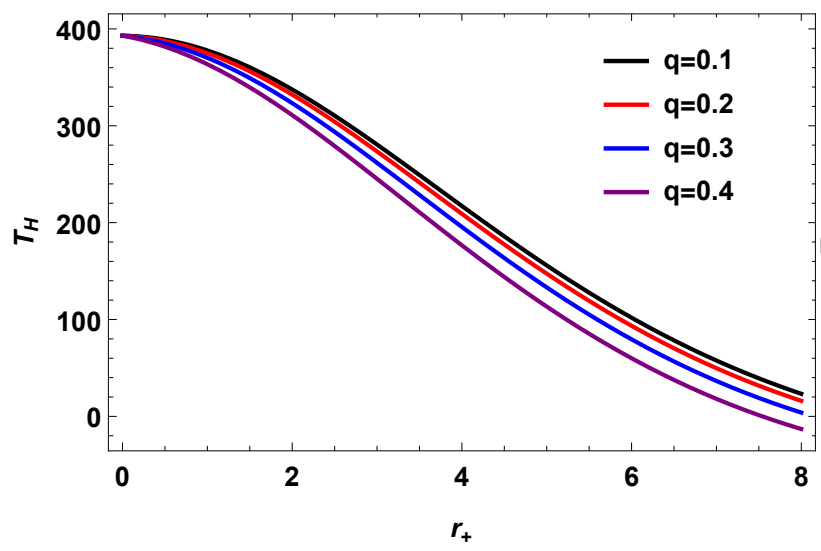

(ii) $M=200, \beta=-0.00005, q=0.1$

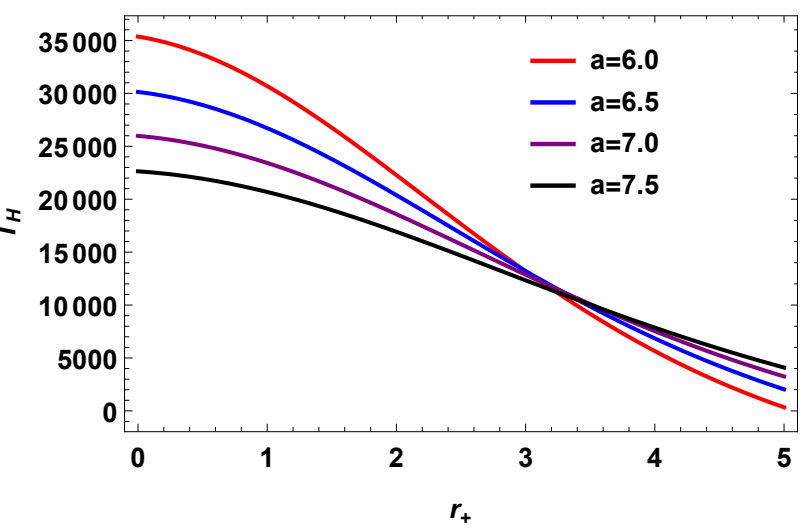

Figure 1: Hawking temperature $T_{H}$ versus event horizon $r_{+}$.

\section{CORRECTED TEMPERATURE FOR CHARGED BLACK STRINGS IN RASTALL THEORY}

This section analyzes the quantum gravity effects on Hawking temperature of charged black strings in the Rastall theory for massive vector particles. To do so, we write the Eq. (15) in the following form

$$
d s^{2}=-A d t^{2}+B d r^{2}+C d \theta^{2}+D d y^{2}+2 E d t d y,
$$

where

$$
\begin{aligned}
& A=\left(\frac{\beta^{2} r^{4}-\frac{4 M r}{\beta}+\frac{4 q^{2}}{\beta^{2}}}{\Sigma^{2}}\right), \quad D=\frac{a^{2}\left[\Sigma^{4}+a^{2}\left(-4 q^{2}+\left(4 M r-r^{4} \beta^{3}+2 \beta \Sigma^{2}\right) \beta\right)\right]}{\Sigma^{2}}, \\
& B=\frac{\Sigma^{2}}{\Delta_{r}}, \quad C=\Sigma^{2}, \quad E=-a \beta^{2}\left(1-\frac{\beta^{2} r^{4}-\frac{4 M r}{\beta}+\frac{4 q^{2}}{\beta^{2}}}{\Sigma^{2}}\right) .
\end{aligned}
$$


The equation of wave motion is defined as [48]

$$
\begin{aligned}
& \partial_{\mu}\left(\sqrt{-g} \chi^{v \mu}\right)+\sqrt{-g} \frac{m^{2}}{\hbar^{2}} \chi^{v}+\sqrt{-g} \frac{i}{\hbar} A_{\mu} \chi^{\nu \mu}+\sqrt{-g} \frac{i}{\hbar} e F^{v \mu} \chi_{\mu}+\alpha \hbar^{2} \partial_{0} \partial_{0} \partial_{0}\left(\sqrt{-g} g^{00} \chi^{0 v}\right) \\
& -\alpha \hbar^{2} \partial_{i} \partial_{i} \partial_{i}\left(\sqrt{-g} g^{i i} \chi^{i v}\right)=0,
\end{aligned}
$$

here $g$ gives the determinant of coefficient matrix, $\chi^{\nu \mu}$ represents the anti-symmetric tensor and $m$ is the particle mass, since

$$
\begin{aligned}
\chi_{v \mu} & =\left(1-\alpha \hbar^{2} \partial_{v}^{2}\right) \partial_{\nu} \chi_{\mu}-\left(1-\alpha \hbar^{2} \partial_{\mu}^{2}\right) \partial_{\mu} \chi_{v}+\left(1-\alpha \hbar^{2} \partial_{v}^{2}\right) \frac{i}{\hbar} e A_{v} \chi_{\mu}-\left(1-\alpha \hbar^{2} \partial_{v}^{2}\right) \frac{i}{\hbar} e A_{\mu} \chi_{v}, \\
F_{v \mu} & =\nabla_{v} A_{\mu}-\nabla_{\mu} A_{\nu},
\end{aligned}
$$

where $\alpha, A_{\mu}, e$ and $\nabla_{\mu}$ represents the dimensionless positive parameter, vector potential, the charge of particle and covariant derivatives, respectively. The non-zero components of anti-symmetric tensor can be computed as

$$
\begin{aligned}
& \chi^{0}=\frac{-D \chi_{0}+E \chi_{3}}{A D+E^{2}}, \quad \chi^{1}=\frac{1}{B} \chi_{1}, \quad \chi^{2}=\frac{1}{C} \chi_{2}, \quad \chi^{3}=\frac{E \chi_{0}+A \chi_{3}}{A D+E^{2}}, \chi^{12}=\frac{1}{B C} \chi_{12}, \chi^{13}=\frac{1}{B A D+E^{2}} \chi_{13}, \\
& \chi^{01}=\frac{-D \chi_{01}+E \chi_{13}}{B\left(A D+E^{2}\right)}, \quad \chi^{02}=\frac{-D \chi_{02}}{C\left(A D+E^{2}\right)}, \quad \chi^{03}=\frac{\left(-A D+A^{2}\right) \chi_{03}}{\left(A D+E^{2}\right)^{2}}, \quad \chi^{23}=\frac{E \chi_{02}+A \chi_{23}}{C\left(A D+E^{2}\right)},
\end{aligned}
$$

The WKB approximation is defined as

$$
\chi_{v}=c_{v} \exp \left[\frac{i}{\hbar} \Theta(t, r, \theta, \phi)\right]
$$

where

$$
\Theta(t, r, \theta, \phi)=\Theta_{0}(t, r, \theta, \phi)+\hbar \Theta_{1}(t, r, \theta, \phi)+\hbar^{2} \Theta_{2}(t, r, \theta, \phi)+\ldots
$$

By neglecting the higher order terms and after substituting all the values in Eq. (19), we obtain the set of wave equations as

$$
\begin{aligned}
& +\frac{D}{B\left(A D+E^{2}\right)}\left[c_{1}\left(\partial_{0} \Theta_{0}\right)\left(\partial_{1} \Theta_{0}\right)+\alpha c_{1}\left(\partial_{0} \Theta_{0}\right)^{3}\left(\partial_{1} \Theta_{0}\right)-c_{0}\left(\partial_{1} \Theta_{0}\right)^{2}-\alpha c_{0}\left(\partial_{1} \Theta_{0}\right)^{4}+c_{1} e A_{0}\left(\partial_{1} \Theta_{0}\right)\right. \\
& \left.+c_{1} \alpha e A_{0}\left(\partial_{0} \Theta_{0}\right)^{2}\left(\partial_{1} \Theta_{0}\right)\right]-\frac{E}{B\left(A D+E^{2}\right)}\left[c_{3}\left(\partial_{1} \Theta_{0}\right)^{2}+\alpha c_{3}\left(\partial_{1} \Theta_{0}\right)^{4}-c_{1}\left(\partial_{1} \Theta_{0}\right)\left(\partial_{3} \Theta_{0}\right)-\alpha c_{1}\left(\partial_{1} \Theta_{0}\right)\left(\partial_{3} \Theta_{0}\right)^{2}\right] \\
& +\frac{D}{C\left(A D+E^{2}\right)}\left[c_{2}\left(\partial_{0} \Theta_{0}\right)\left(\partial_{2} \Theta_{0}\right)+\alpha c_{2}\left(\partial_{0} \Theta_{0}\right)^{3}\left(\partial_{2} \Theta_{0}\right)-c_{0}\left(\partial_{2} \Theta_{0}\right)^{2}-\alpha c_{0}\left(\partial_{2} \Theta_{0}\right)^{4}+c_{2} e A_{0}\left(\partial_{2} \Theta_{0}\right)\right. \\
& \left.+c_{2} e A_{0} \alpha\left(\partial_{0} \Theta_{0}\right)^{2}\left(\partial_{1} \Theta_{0}\right)\right]+\frac{A D}{\left(A D+E^{2}\right)^{2}}\left[c_{3}\left(\partial_{0} \Theta_{0}\right)\left(\partial_{3} \Theta_{0}\right)+\alpha c_{3}\left(\partial_{0} \Theta_{0}\right)^{3}\left(\partial_{3} \Theta_{0}\right)-c_{0}\left(\partial_{3} \Theta_{0}\right)^{2}\right. \\
& \left.-\alpha c_{0}\left(\partial_{3} \Theta_{0}\right)^{4}+c_{3} e A_{0}\left(\partial_{3} \Theta_{0}\right)+c_{3} e A_{0}\left(\partial_{0} \Theta_{0}\right)^{2}\left(\partial_{3} \Theta_{0}\right)\right]-m^{2} \frac{\tilde{D c_{0}}-\tilde{E c_{3}}}{\left(A D+E^{2}\right)}=0, \\
& -\frac{D}{B\left(A D+E^{2}\right)}\left[c_{1}\left(\partial_{0} \Theta_{0}\right)^{2}+\alpha c_{1}\left(\partial_{0} \Theta_{0}\right)^{4}-c_{0}\left(\partial_{0} \Theta_{0}\right)\left(\partial_{1} \Theta_{0}\right)-\alpha c_{0}\left(\partial_{0} \Theta_{0}\right)\left(\partial_{1} \Theta_{0}\right)^{3}+c_{1} e A_{0}\left(\partial_{0} \Theta_{0}\right)\right. \\
& \left.+\alpha c_{1} e A_{0}\left(\partial_{0} \Theta_{0}\right)^{3}\right]+\frac{E}{B\left(A D+E^{2}\right)}\left[c_{3}\left(\partial_{0} \Theta_{0}\right)\left(\partial_{1} \Theta_{0}\right)+\alpha c_{3}\left(\partial_{0} \Theta_{0}\right)\left(\partial_{1} \Theta_{0}\right)^{3}-c_{1}\left(\partial_{0} \Theta_{0}\right)\left(\partial_{3} \Theta_{0}\right)-\alpha c_{1}\left(\partial_{0} \Theta_{0}\right)\left(\partial_{3} \Theta_{0}\right)^{3}\right] \\
& +\frac{1}{B C}\left[c_{2}\left(\partial_{1} \Theta_{0}\right)\left(\partial_{2} \Theta_{0}\right)+\alpha c_{2}\left(\partial_{1} \Theta_{0}\right)\left(\partial_{2} \Theta_{0}\right)^{3}-c_{1}\left(\partial_{2} \Theta_{0}\right)^{2}-\alpha c_{1}\left(\partial_{2} \Theta_{0}\right)^{4}\right]+\frac{1}{B\left(A D+E^{2}\right)}\left[c_{3}\left(\partial_{1} \Theta_{0}\right)\left(\partial_{3} \Theta_{0}\right)+\alpha c_{3}\right. \\
& \left.\times\left(\partial_{1} \Theta_{0}\right)\left(\partial_{3} \Theta_{0}\right)^{3}-c_{1}\left(\partial_{3} \Theta_{0}\right)^{2}-\alpha c_{1}\left(\partial_{3} \Theta_{0}\right)^{4}\right]+\frac{e A_{0} D}{B\left(A D+E^{2}\right)}\left[c_{1}\left(\partial_{0} \Theta_{0}\right)+\alpha c_{1}\left(\partial_{0} \Theta_{0}\right)^{3}-c_{0}\left(\partial_{1} \Theta_{0}\right)-\alpha c_{0}\left(\partial_{1} \Theta_{0}\right)^{3}\right. \\
& \left.\left.+e A_{0} c_{1}+\alpha c_{1} e A_{0}\left(\partial_{0} \Theta_{0}\right)^{2}\right)\right]+\frac{e A_{0} E}{B\left(A D+E^{2}\right)}\left[c_{3}\left(\partial_{1} \Theta_{0}\right)+\alpha c_{3}\left(\partial_{1} \Theta_{0}\right)^{3}-c_{1}\left(\partial_{3} \Theta_{0}\right)-\alpha c_{1}\left(\partial_{1} \Theta_{0}\right)^{3}\right]-\frac{m^{2} c_{1}}{B}=0,
\end{aligned}
$$




$$
\begin{aligned}
& +\frac{D}{C\left(A D+E^{2}\right)}\left[c_{2}\left(\partial_{0} \Theta_{0}\right)^{2}+\alpha c_{2}\left(\partial_{0} \Theta_{0}\right)^{4}-c_{0}\left(\partial_{0} \Theta_{0}\right)\left(\partial_{2} \Theta_{0}\right)-\alpha c_{0}\left(\partial_{0} \Theta_{0}\right)\left(\partial_{2} \Theta_{0}\right)^{3}+c_{2} e A_{0}\left(\partial_{0} \Theta_{0}\right)+\alpha c_{2} e A_{0}\left(\partial_{0} \Theta_{0}\right)^{3}\right] \\
& +\frac{1}{B C}\left[c_{2}\left(\partial_{1} \Theta_{0}\right)^{2}+\alpha c_{2}\left(\partial_{1} \Theta_{0}\right)^{4}-c_{1}\left(\partial_{1} \Theta_{0}\right)\left(\partial_{2} \Theta_{0}\right)-\alpha c_{1}\left(\partial_{1} \Theta_{0}\right)\left(\partial_{2} \Theta_{0}\right)^{3}\right]-\frac{E}{C\left(A D+E^{2}\right)}\left[c_{2}\left(\partial_{0} \Theta_{0}\right)\left(\partial_{3} \Theta_{0}\right)\right. \\
& \left.+\alpha c_{2}\left(\partial_{0} \Theta_{0}\right)^{3}\left(\partial_{3} \Theta_{0}\right)-c_{0}\left(\partial_{0} \Theta_{0}\right)\left(\partial_{3} \Theta_{0}\right)-\alpha c_{0}\left(\partial_{0} \Theta_{0}\right)^{3}\left(\partial_{3} \Theta_{0}\right)+c_{2} e A_{0}\left(\partial_{3} \Theta_{0}\right)+\alpha c_{2} e A_{0}\left(\partial_{3} \Theta_{0}\right)^{3}\right] \\
& +\frac{A}{C\left(A D+E^{2}\right)}\left[c_{3}\left(\partial_{2} \Theta_{0}\right)\left(\partial_{3} \Theta_{0}\right)+\alpha c_{3}\left(\partial_{2} \Theta_{0}\right)^{3}\left(\partial_{3} \Theta_{0}\right)-c_{2}\left(\partial_{3} \Theta_{0}\right)^{2}-\alpha c_{2}\left(\partial_{3} \Theta_{0}\right)^{4}\right]-\frac{m^{2} c_{2}}{C} \\
& +\frac{e A_{0} D}{C\left(A D+E^{2}\right)}\left[c_{2}\left(\partial_{0} \Theta_{0}\right)+\alpha c_{2}\left(\partial_{0} \Theta_{0}\right)^{3}-c_{0}\left(\partial_{2} \Theta_{0}\right)-\alpha c_{0}\left(\partial_{2} \Theta_{0}\right)^{3}+c_{2} e A_{0}+c_{2} \alpha e A_{0}\left(\partial_{0} \Theta_{0}\right)^{2}\right]=0, \\
& +\frac{(A D)-A^{2}}{\left(A D+E^{2}\right)^{2}}\left[c_{3}\left(\partial_{0} \Theta_{0}\right)^{2}+\alpha c_{3}\left(\partial_{0} \Theta_{0}\right)^{4}-c_{0}\left(\partial_{0} \Theta_{0}\right)\left(\partial_{3} \Theta_{0}\right)-\alpha c_{0}\left(\partial_{0} \Theta_{0}\right)\left(\partial_{3} \Theta_{0}\right)^{3}+e A_{0} c_{3}\left(\partial_{0} \Theta_{0}\right)\right. \\
& \left.+\alpha c_{3} e A_{0}\left(\partial_{0} \Theta_{0}\right)^{3}\right]-\frac{D}{C\left(A D+E^{2}\right)}\left[c_{3}\left(\partial_{1} \Theta_{0}\right)^{2}+\alpha c_{3}\left(\partial_{1} \Theta_{0}\right)^{4}-c_{1}\left(\partial_{1} \Theta_{0}\right)\left(\partial_{3} \Theta_{0}\right)-\alpha c_{1}\left(\partial_{1} \Theta_{0}\right)\left(\partial_{3} \Theta_{0}\right)^{3}\right] \\
& -\frac{E}{C\left(A D+E^{2}\right)}\left[c_{2}\left(\partial_{0} \Theta_{0}\right)\left(\partial_{2} \Theta_{0}\right)+\alpha c_{2}\left(\partial_{0} \Theta_{0}\right)^{3}\left(\partial_{2} \Theta_{0}\right)-c_{0}\left(\partial_{2} \Theta_{0}\right)^{2}+\alpha c_{0}\left(\partial_{2} \Theta_{0}\right)^{4}+e A_{0} c_{2}\left(\partial_{2} \Theta_{0}\right)+\alpha c_{2} e A_{0}\right. \\
& \left.\times\left(\partial_{0} \Theta_{0}\right)^{2}\left(\partial_{2} \Theta_{0}\right)\right]-\frac{e A_{0} A}{C\left(A D+E^{2}\right)}\left[c_{3}\left(\partial_{2} \Theta_{0}\right)^{2}+\alpha c_{3}\left(\partial_{2} \Theta_{0}\right)^{4}-c_{2}\left(\partial_{2} \Theta_{0}\right)\left(\partial_{3} \Theta_{0}\right)-\alpha c_{2}\left(\partial_{0} \Theta_{0}\right)\left(\partial_{3} \Theta_{0}\right)^{3}\right] \\
& +\frac{e A_{0}(A D)-A^{2}}{\left(A D+E^{2}\right)^{2}}\left[c_{3}\left(\partial_{0} \Theta_{0}\right)+\alpha c_{3}\left(\partial_{0} \Theta_{0}\right)^{3}-c_{0}\left(\partial_{3} \Theta_{0}\right)-\alpha c_{0}\left(\partial_{3} \Theta_{0}\right)^{3}+c_{3} e A_{0}+\alpha e A_{0}\left(\partial_{0} \Theta_{0}\right)^{2}\right] \\
& -\frac{m^{2}\left(E c_{0}-A c_{3}\right.}{\left(A D+E^{2}\right)}=0
\end{aligned}
$$

Using separation of variables technique, we can choose

$$
\Theta_{0}=-\hat{E} t+W(r)+J \phi+v(\theta),
$$

where $E=(E-j \omega), E$ denotes the energy of the particle, $J$ represents the particles angular momentum corresponding to angles $\phi$. After substituting Eq. (26) into set of wave equations, we get a $4 \times 4$ matrix

$$
\mathcal{Z}\left(c_{0}, c_{1}, c_{2}, c_{3}\right)^{T}=0
$$

whose components are given as follows:

$$
\begin{aligned}
& \mathcal{Z}_{00}=\frac{-\tilde{D}}{B\left(A D+E^{2}\right)}\left[W_{1}^{2}+\alpha W_{1}^{4}\right]-\frac{D}{C\left(A D+E^{2}\right)}\left[J^{2}+\alpha J^{4}\right],-\frac{A D}{\left(A D+E^{2}\right)^{2}}\left[v_{1}^{2}+\alpha v_{1}^{4}\right]-\frac{m^{2} D}{\left(A D+E^{2}\right)^{\prime}} \\
& \mathcal{Z}_{01}=\frac{\tilde{D}}{B\left(A D+E^{2}\right)}\left[\hat{E}+\alpha \hat{E}^{3}+e A_{0}+\alpha e A_{0} \hat{E}^{2}\right] W_{1}+\frac{E}{B\left(A D+E^{2}\right)}+\left[v_{1}+\alpha v_{1}^{3}\right] \text {, } \\
& \mathcal{Z}_{02}=\frac{\tilde{-D}}{C\left(A D+E^{2}\right)}\left[\hat{E}+\alpha \hat{E}^{3}-e A_{0}-\alpha e A_{0} \hat{E}^{2}\right] J, \\
& \mathcal{Z}_{03}=\frac{\tilde{-E}}{B\left(A D+E^{2}\right)}\left[W_{1}^{2}+\alpha W_{1}^{4}\right]-\frac{A D}{C\left(A D+E^{2}\right)^{2}}\left[\hat{E}+\alpha E^{3}-e A_{0}-\alpha e A_{0} \dot{E}^{2}\right] v_{1}+\frac{m^{2} E}{\left(A D+E^{2}\right)^{2}}, \\
& \mathcal{Z}_{11}=\frac{-\tilde{D}}{B\left(A D+E^{2}\right)}\left[\dot{E}^{2}+\alpha \dot{E}^{4}-e A_{0} \dot{E}-\alpha e A_{0} E^{\prime} W_{1}^{2}\right]+\frac{E}{B\left(A D+E^{2}\right)}-\frac{m^{2}}{B} \\
& +\left[v_{1}+\alpha v_{1}^{3}\right] \hat{E}-\frac{1}{B C}\left[J^{2}+\alpha J^{4}\right]-\frac{1}{B\left(A D+E^{2}\right)}\left[v_{1}+\alpha v_{1}^{3}\right]+\frac{e A_{0} E}{B\left(A D+E^{2}\right)}\left[v_{1}+\alpha v_{1}^{3}\right] \\
& -\frac{e A_{0} D}{B\left(A D+E^{2}\right)}\left[\hat{E}+\alpha \hat{E}^{3}-e A_{0}-\alpha e A_{0} E^{2}\right], \quad \mathcal{Z}_{12}=\frac{1}{B C}\left[W_{1}+\alpha W_{1}^{3}\right] J, \\
& \mathcal{Z}_{13}=\frac{\tilde{\tau_{E}}}{B\left(A D+E^{2}\right)}\left[W_{1}+\alpha W_{1}^{3}\right] \hat{E}+\frac{1}{B\left(A D+E^{2}\right)^{2}}\left[W_{1}+\alpha W_{1}^{3}\right] v_{1}+\frac{E e A_{0}}{B\left(A D+E^{2}\right)}\left[W_{1}+\alpha W_{1}^{3}\right] \text {, }
\end{aligned}
$$




$$
\begin{aligned}
\mathcal{Z}_{22} & =\frac{D}{C\left(A D+E^{2}\right)}\left[\hat{E}^{2}+\alpha \hat{E}^{4}-e A_{0} \hat{E}-\alpha e A_{0} \dot{E}\right]-\frac{1}{B C}-\frac{m^{2}}{C} \\
& -\frac{A}{C\left(A D+E^{2}\right)}\left[v_{1}^{2}+\alpha v_{1}^{4}\right]-\frac{e A_{0} D}{C\left(A D+E^{2}\right)}\left[\hat{E}+\alpha \hat{E}^{3}-e A_{0}-\alpha e A_{0} \dot{E}^{2}\right] \\
& +\frac{E}{C\left(A D+E^{2}\right)}\left[\hat{E}+\alpha \hat{E}^{3}-e A_{0}-\alpha e A_{0} \hat{E}^{2}\right] v_{1}, \\
\mathcal{Z}_{23} & =\frac{A}{C\left(A D+E^{2}\right)}\left[J+\alpha J^{3}\right] v_{1}, \quad \mathcal{Z}_{31}=\frac{1}{B\left(A D+E^{2}\right)}\left[v_{1}+\alpha v_{1}^{3}\right] W_{1}, \\
\mathcal{Z}_{33} & =\frac{\left(A D-\tilde{A}^{2}\right)}{\left(A D+E^{2}\right)}\left[\hat{E}^{2}+\alpha \hat{E}^{4}-e A_{0} \hat{E}-\alpha e A_{0} \dot{E}^{3}\right]-\frac{1}{B\left(A D+E^{2}\right)}\left[W_{1}^{2}+\alpha W_{1}^{4}\right] \\
& -\frac{A}{C\left(A D+E^{2}\right)}\left[J^{2}+\alpha J^{4}\right]-\frac{m^{2} A}{\left(A D+E^{2}\right)}-\frac{e A_{0}\left(A D-\tilde{A}^{2}\right)}{\left(A D+E^{2}\right)}\left[\hat{E}+\alpha \hat{E}^{3}-e A_{0} \dot{E}^{2}\right],
\end{aligned}
$$

where $J=\partial_{\phi} \Theta_{0}, W_{1}=\partial_{r} \Theta_{0}$ and $v_{1}=\partial_{\theta} \Theta_{0}$. For the non-trivial solution, we set determinant $\mathcal{Z}$ is equal to zero and get

$$
\begin{aligned}
\operatorname{Im} W^{ \pm} & = \pm \int \sqrt{\frac{\left(\hat{E}-e A_{0}\right)^{2}+X_{1}\left[1+\alpha \frac{X_{2}}{X_{1}}\right]}{\left(A D+E^{2}\right) / B D}} d r \\
& = \pm \pi \frac{\left(\dot{E}-e A_{0}\right)+[1+\alpha \Xi]}{2 \kappa\left(r_{+}\right)}
\end{aligned}
$$

where

$$
\begin{aligned}
X_{1} & =\frac{B E}{\left(A D+E^{2}\right)}\left[\dot{E}-e A_{0}\right] v_{1}+\frac{A B}{\left(A D+E^{2}\right)} v_{1}^{2}-B m^{2}, \\
X_{2} & =\frac{B D}{\left(A D+E^{2}\right)}\left[\dot{E}^{4}-2 e A_{0} \dot{E}^{3}+\left(e A_{0}\right)^{2} \dot{E}^{2}\right]-\frac{A B}{\left(A D+E^{2}\right)} v_{1}^{4}-W_{1}^{4} \\
& +\frac{B E}{C\left(A D+E^{2}\right)}\left[\dot{E}^{3}-e A_{0} \dot{E}^{2}\right] v_{1} .
\end{aligned}
$$

The tunneling probability for charged vector particles can be given as

$$
\Gamma=\frac{\Gamma_{\text {emission }}}{\Gamma_{\text {absorption }}}=\exp \left[-2 \pi \frac{\left(\dot{E}-e A_{0}\right)}{\kappa\left(r_{+}\right)}\right][1+\alpha \Xi] .
$$

where

$$
\kappa\left(r_{+}\right)=\frac{2 \beta^{4} r_{+}^{5}+4 M \beta r_{+}^{2}-8 r_{+} q^{2}+a^{2}\left(4 \beta^{4} r_{+}^{3}-4 M \beta\right)}{2 \alpha^{2}\left(r_{+}^{2}+a^{2}\right)^{2}} .
$$

The modified Hawking temperature can be derived after expanding the series $[1+\alpha \Xi]$ and by using the Boltzmann factor $\Gamma_{B}=\exp \left[\left(E-e A_{0}\right) / T_{H}^{\prime}\right]$ as

$$
T_{H}^{\prime} \cong \frac{2 \beta^{4} r_{+}^{5}+4 M \beta r_{+}^{2}-8 r_{+} q^{2} a^{2}\left(4 \beta^{4} r_{+}^{3}-4 M \beta\right)}{4 \pi \alpha^{2}\left(r_{+}^{2}+a^{2}\right)^{2}}[1-\alpha \Xi]
$$

The modified Hawking temperature of charged black strings depends upon quantum gravity parameter $\alpha$, mass $M$, charge $q$, spin parameter $a$ and cosmological constant $\Lambda$ (i. e., $\beta=-\Lambda / 3$ ). In the absence of quantum gravity parameter $\alpha=0$, we observe the temperature of Eq. (16).

\section{GRAPHICAL ANALYSIS}

The section comprises the graphical behavior of modified temperature of charged black strings in Rastall theory. We examine the effects of quantum gravity parameters $\alpha$ and spin parameter $a$ (appears due to Rastall theory) from 
charged black strings. We also study the physical significance of these plots. These plots depicts the behavior of $T_{H}^{\prime}$ w.r.t horizon $r_{+}$.

In Fig. 2: (i) indicates the behavior of $T_{H}^{\prime}$ for fixed values of $M=100, \beta=0.01, q=1, a=9, \Xi=10$ and various values of quantum gravity parameter $\alpha$ in the range $0 \leq r_{+} \leq 8$. One can observe that $T_{H}^{\prime}$ goes on decreasing for increasing values of $r_{+}$. This is physical phenomenon and represents the stable condition of charged black strings under the influence of quantum gravity parameter at high temperature.

(ii) shows the behavior of $T_{H}^{\prime}$ for fixed $M=100, \beta=0.01, q=8, \alpha=500, \Xi=10$ and various values of spin parameter $a$. One can see that at first the $T_{H}^{\prime}$ increases very slowly and it reaches at a maximum height with very high value and the eventually falls down from height and attains an asymptotically flat form as $T_{H}^{\prime} \rightarrow 0$ till $r_{+} \rightarrow \infty$. This is purely stable and physical form of charged black strings under the effects of spin parameter and quantum gravity. It is notable that as we increase the values of spin parameter the temperature decreases. Moreover, the very high $T_{H}^{\prime}$ at non-zero $r_{+}$indicates the $\mathrm{BH}$ remnant.

(i) $M=100, \beta=0.01, q=1, a=9, \equiv=10$

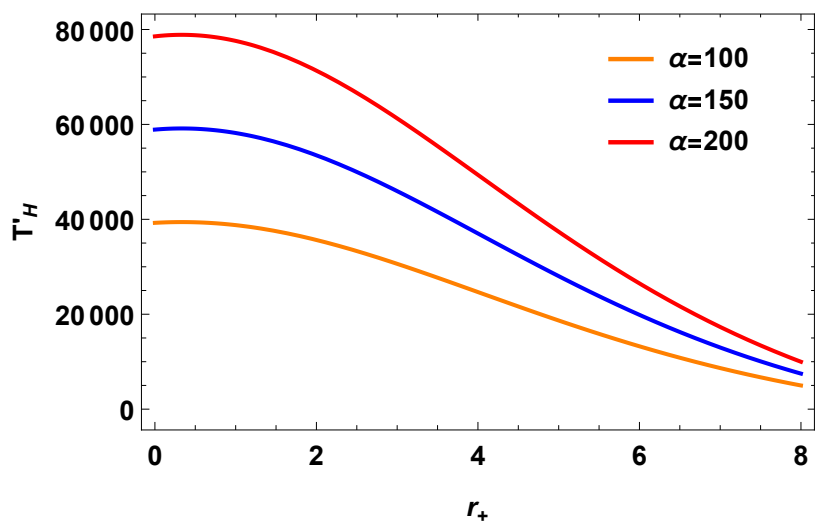

(ii) $M=100, \beta=0.01, q=8, \alpha=500, \equiv=10$

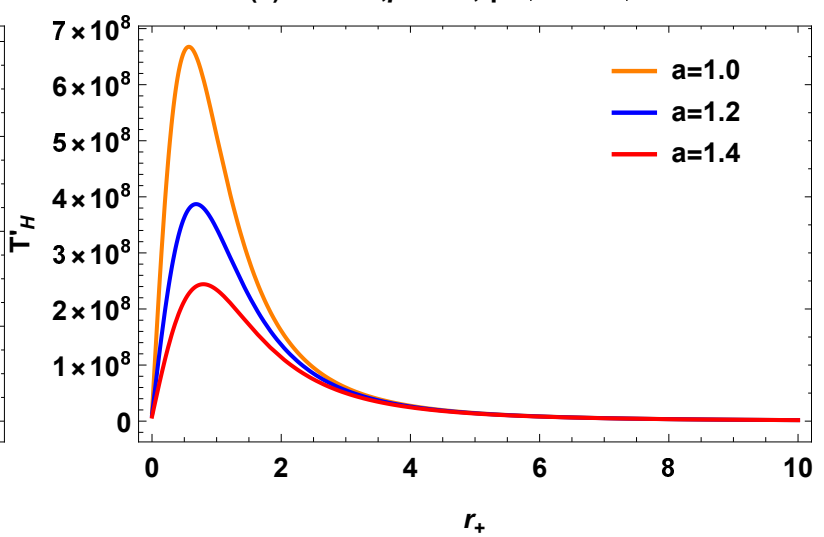

Figure 2: Hawking temperature $T_{H}^{\prime}$ versus event horizon $r_{+}$.

\section{SUMMARY AND DISCUSSION}

In our work, we investigated the charged black strings solution in the context of Rastall theory by applying the Newman-Janis algorithm. After assuming the spin parameter $(a \rightarrow 0)$ in the Eq. (15), we obtained the black strings solution without Rastall theory in general relativity. The charged black strings solution in Rastall theory is quite different from the $\mathrm{BH}$ solution in general theory of relativity.

The Hawking temperature $T_{H}$ depends on cosmological constant $\Lambda$, spin parameter $a$, black string mass $M$ and black string charge $q$. It is worth mentioning here that for $a=0$, we recovered the Hawking temperature for charged black strings [50] that is independent of the spin parameter. It is suggested that the back-reaction affects of the emitted particles on the black string geometry as well as self-gravitating impacts have been neglected and evaluated Hawking temperature as a term and yields as black string geometry. The Hawking radiation from the charged black strings have different types of particles spins (down, upward or zero spin). In this procedure, the Hawking temperature is associated to the spin parameter and geometry of charged black strings. We conclude from the graphical interpretation of temperature $T_{H}$ w.r.t horizon $r_{+}$that the charged black strings solution under the influence of Rastall theory for various values of charge and spin parameter depicts its stable form. Furthermore, we examined the quantum gravity effects for charged black strings in Rastall theory and derived the modified Hawking temperature. We also discussed the stable and physical form of charged black strings under the effects of quantum gravity and spin parameter. The spin parameter which appears due to Rastall theory in charged black string solution causes the reduction in temperature. The Hawking's phenomenon depicts that with the emission of more radiations the size of $\mathrm{BH}$ radius reduces and we observe $\mathrm{BH}$ remnant at very high temperature with non-zero horizon. We observe this physical phenomenon in all plots which guarantee the stable form of charged black strings. Since, the conclusion still holds if background charged black strings geometry is more general.

[1] P. Rastall, Phys. Rev. D 6, 3357(1972).

[2] P. Rastall, Can. J. Phys. 54, 66(1976). 
[3] Y. Heydarzade, F. Darabi, Phys. Lett. B 771, 365(2017).

[4] A. M. Oliveira, H. E. S. Velten, J. C. Fabris, L. Casarini, Phys. Rev. D 92, 044020(2015).

[5] H. Moradpour, N. Sadeghnezhad, Can. J. Phys. 95, 1257(2017).

[6] Y. Heydarzade, H. Moradpour, F. Darabi, Can. J. Phys. 95, 1253(2017).

[7] E. Spallucci, A. Smailagic, Int. J. Mod. Phys. D 27, 1850003(2018).

[8] R. Kumar, S. G. Ghosh, Eur. Phys. J. C 78, 750(2018).

[9] Z. Xu, X. Hou, X. Gong, J. Wang, Eur. Phys. J. C 78, 513(2018).

[10] K. Lin, W. L. Qian, Chinese Phys. C 43, 083106(2019).

[11] M. Visser, Phys. Lett. B 782, 83(2018).

[12] F. Darabi, H. Moradpour, I. Licata, Y. Heydarzade, C. Corda, Eur. Phys. J. C 78, 25(2018).

[13] M. F. A. R. Sakti, A. Suroso, F. P. Zen, Ann. Phys. 413, 168062(2020).

[14] H. Moradpour, Y. Heydarzade, C. Corda, A. H. Ziaie, S. Ghaffari, Mod. Phys. Lett. A 34, 1950304(2019).

[15] A. Yale, Phys. Lett. B 697, 398(2011).

[16] W. Javed, G. Abbas, R. Ali, Eur. Phys. J. C 77, 296(2017).

[17] W. Javed, R. Babar, Adv. High Energy Phys. 2019, 2759641(2019); ibid. Chinese Journal of Phys. 61, 138(2019); Proceedings of the 15th Marcel Grossmann Meeting, http:/ / robot.icranet.org:8080/store/1380.pdf; ibid. Punjab University Journal of Mathematics 52, 6(2020).

[18] W. Javed, R. Babar, A. Övgün, Mod. Phys. Lett. A 34, 1950057(2019).

[19] R. Babar, W. Javed, A. Övgün, Mod. Phys. Lett. A 35, 2050104(2020).

[20] M. Sharif, W. Javed, Can. J. Phys. 90, 903(2012); ibid. Gen. Relativ. Gravit. 45, 1051(2013); ibid. Can. J. Phys. 91, 43(2013); ibid. J. Exp. Theor. Phys. 115, 782(2012); ibid. Proceedings of the 3rd Galileo-Xu Guangqi Meeting, Int. J. Mod. Phys.: Conference Series, 23, 271(2013);ibid. Proceedings of the 13th Marcel Grossmann Meeting (Stockholm, 2012), World Scientific, 3, 1950(2015).

[21] M. Sharif, W. Javed, Eur. Phys. J. C 72, 1997(2012).

[22] M. Sharif, W. Javed, J. Korean Phys. Soc. 57, 217(2010).

[23] A. Övgün, K. Jusufi, Eur. Phys. J. Plus. 132, 298(2017).

[24] X. Q. Li, G.R. Chen, Phys. Lett. B 751, 34(2015).

[25] W. Javed, R. Ali, G. Abbas, Can. J. Phys. 97, 176(2018).

[26] A. Övgün, W. Javed, R. Ali, Adv. High Energy Phys. 2018, 11(2018).

[27] A. Övgün, Int. J. Theor. Phys. 55, 2919(2016).

[28] A. Övgün, K. Jusufi, Eur. Phys. J. Plus 132, 298(2017).

[29] K. Jusufi, A. Ovgun, G. Apostolovska, Adv. High Energy Phys. 2017, 8798657(2017).

[30] R. Casadio, P. Nicolini, R. da Rocha, Class. Quantum Grav. 35, 185001(2018).

[31] S. Kanzi, I. Sakalli, Nucl. Phys. B 946, 114703(2019).

[32] Y. K. Meitei, T. I. Singh, I. A. Meitei, Turk. J. Phys. 44, 373(2020).

[33] I. Sakalli, A. Övgün, K. Jusufi, Astrophys Space Sci. 361, 330(2016).

[34] G. Gecim, Y. Sucu, Phys. Lett. B 773, 391(2017).

[35] I. Sakalli, A. Övgün, General Relativity and Gravitation 48, 1(2016).

[36] A. Övgün, I. Sakalli, Int. J. Theor. Phys. 57, 322(2018).

[37] G. Abbas, M. R. Shahzad, Chinese J. Phys. 63, 1(2020).

[38] A. Övgün, I. Sakalli, J. Saavedra, C. Leiva, Mod. Phys. Lett. A 35, 2050163(2020).

[39] X. C. Cai, Y. G. Miao, Phys. Rev. D 101, 104023(2020).

[40] I. Sakalli, A. Ovgun, J. Exp. Theor. Phys. 121, 404(2015).

[41] J. M. Bardeen, in Conference Proceedings of GR5 (Tbilisi, URSS, 1968), p. 174 J. M. Bardeen, in Conference Proceedings of GR5 (Tbilisi, URSS, 1968), p. 174.

[42] D. Y. Chen, H. W. Wu, H. T. Yang, J. Cosmol. Astropart. Phys. 03, 036(2014).

[43] D. Chen, H. Wu, H. Yang, Adv. High Energy Phys. 2013, 432412(2013).

[44] R. Ali, K. Bamba, S. A. A. Shah, Symmetry. 631, 11(2019).

[45] R. Ali, K. Bamba, M. Asgher, M. F. Malik, S. A. A. Shah, Symmetry. 12, 1165(2020).

[46] R. Ali, K. Bamba, M. Asgher, S. A. A. Shah, Int. J. Mod. Phys. D 30, 2150002(2021).

[47] R. Ali, M. Asgher, M. F. Malik, Mod. Phys. Lett. A 35, 2050225(2020).

[48] W. Javed, R. Ali, R. Babar, A. Övgün, Eur. Phys. J. Plus 134, 511(2019); ibid. Chinese Phys. C 44, 015104(2020).

[49] M. F. A. R. Sakti, A. Suroso, F. P. Zen, Annals of Phys. 413, 168062(2020).

[50] K. Jusufi, A. Övgün, Astrophys Space Sci. 361, 207(2016).

[51] H. Gohar, K. Saifullah, Astrophys Space Sci. 343, 181(2013). 